Kragujevac Journal of Mathematics

Volume 46(1) (2022), PAges 125-137.

\title{
PSEUDO-BCK ALGEBRAS DERIVED FROM DIRECTOIDS
}

\begin{abstract}
AKBAR REZAEI ${ }^{1}$
ABSTRACT. The aim of this paper is to derive pseudo-BCK algebras from directoids and vice versa. We generalize some results proved by Ivan Chajda et al. in the case of BCK-algebras. We assign to an arbitrary pseudo-BCK algebra a semilattice-like structure and observe that this is the point where directoids are different from the semilattice-like structures. Finally, the relation between commutative deductive systems and derive directoids from a bounded pseudo-BCK $(\mathrm{pDN})$ algebras and a characterization of commutative deductive systems of a bounded pseudo-BCK $(\mathrm{pDN})$ algebra in terms of directoids is discussed.
\end{abstract}

\section{INTRODUCTION}

BCK-algebras were introduced by Y. Imai and K. Iséki in 1966 ([15, 19]) as algebras with a binary operation $*$ modeling the set-theoretical difference and with a constant element 0 that is a least element. S. Tanaka defined a special class of BCK-algebras called commutative BCK-algebras in 1975 (see [31]). In BCK-algebras, some lattices, as bounded commutative BCK-algebras, involutive BCK-lattices and bounded implicative BCK-algebras were defined and among the relationship between them were discussed [23]. Some recent researchers led to generalizations of the notion of pseudo structure on some types of algebras. G. Georgescu et al. [10] and independently J. Rachůnek [24], introduced pseudo-MV algebra which is a non-commutative generalization of MV-algebra. After a pseudo-MV algebra, the pseudo-BL algebra [11], the pseudo-BCK algebra [12] and as a generalization of BCI-algebra, the notion of pseudo-BCI algebra is introduced by W. A. Dudek et al. in [9]. A. Walendziak [32] introduced pseudo-BCH algebras as an extension of BCH-algebras. Further, he

Key words and phrases. (Commutative) pseudo-BCK algebra, pseudo-BCK lattice, (commutative) deductive system, directoid, semilattice.

2010 Mathematics Subject Classification. 03G25, 06F35, 03F50.

DOI 10.46793/KgJMat2201.125R

Received: November 30, 2018.

Accepted: September 11, 2019. 
proved that every branchwise commutative pseudo-BCH algebra is a pseudo-BCI algebra [33]. Commutative pseudo-BCK algebras were originally defined by G. Georgescu et al. in [12] under the name of semilattice-ordered pseudo-BCK algebras and some properties of these structures were investigated by J. Kühr in [21,22]. R. A. Borzooei et al. introduced in [1] (see also $[2,26,27]$ ) a pseudo-BE algebra as generalization of $\mathrm{BE}$-algebra, and the commutative pseudo-BE algebra have recently been investigated by L. C. Ciungu. It was proved that the class of commutative pseudo-BE algebras is equivalent to the class of commutative pseudo-BCK algebras. Based on this result, all results holding for commutative pseudo-BCK algebras also hold for commutative pseudo-BE algebras [5]. Then she gave a characterization of commutative pseudo-BCK algebras and defined the commutative deductive systems of pseudo-BCK algebras and proved that a pseudo-BCK algebra $\mathfrak{X}$ is commutative if and only if all the deductive systems of $\mathfrak{X}$ are commutative. Also, she showed that the class of commutative pseudo-BCK algebras is a variety [6] (see also, [14]). A. Rezaei et al. introduced the notion of pseudo-CI algebras as an extension of pseudo-BE algebras and proved that the class of commutative pseudo-CI algebras coincide with the class of commutative pseudo-BCK algebras [28]. G. Georgescu et al. proved that every Wajsberg pseudo-hoop is a basic pseudo-hoop and every simple basic pseudo-hoop is a linearly ordered Wajsberg pseudo-hoop [13]. L. C. Ciungu in [7] showed that every pseudo-hoop is a pseudo-BCK-meet semilattice. The relation between $\mathrm{FL}_{w}$-algebras, bounded pseudo-BCK $(\mathrm{pP})$ algebras, pseudo-MTL algebras, pseudo-BL algebras and pseudo-MV algebras proved in [16]. Also, in [29,30], the interrelationships between dual pseudo-Q/QC algebras and other pseudo algebras are visualized with a diagram and then they introduced the concepts of branchwise commutative pseudo-CI algebras and pointed pseudo-CI algebras and investigated some of properties. A. Iorgulescu for the first time introduced the notation of quasi-pseudo-M algebras as generalizations of pseudo-M algebras and (involutive) quasi-implicative-groups and the (strong involutive) (super) quasi-implicative-hoops, as generalizations of implicative-groups and implicative-hoops, respectively in [18]. I. Chajda et al. showed that one can be assign to an arbitrary BCK-algebra a semilattice-like structure every section of which possesses a certain antitone mappings [3], it arises a natural question of generalization of these concepts also for pseudo-BCK algebras. Since lattice theory has many applications in computer science and has an important and vital role in investigating the structure of a logical system, this motivated our investigations on directoids and pseudo-BCK-algebras to characterized several of its important properties. The main result of this paper establishes a bijective correspondence between pseudo-BCK algebras and some algebraic structures defined by two directoids. A characterization of commutative deductive systems of a bounded pseudo-BCK $(\mathrm{pDN})$ algebra in terms of directoids is discussed and various results obtained mentioned in this paper can be transferred to the pseudo-BCK algebras. The core of the paper is based on by presenting a survey of some results of logic in the non-commutative case (see [3] for the commutative case) and extension of [25] (see also [4]). 


\section{Preliminaries}

In this section we recall some basic notions and results regarding (commutative) pseudo-BCK algebras.

Definition $2.1([9,17])$. An algebra $\mathfrak{X}=(X ; \rightarrow, \rightsquigarrow, 1)$ of type $(2,2,0)$ is called a pseudo-BCI algebra if it satisfies the following axioms for all $x, y, z \in X$ :

$\left(\mathrm{psBCI}_{1}\right)(x \rightarrow y) \rightsquigarrow((y \rightarrow z) \rightsquigarrow(x \rightarrow z))=1$ and $(x \rightsquigarrow y) \rightarrow((y \rightsquigarrow z) \rightarrow(x \rightsquigarrow z))=1 ;$

$\left(\operatorname{psBCI}_{2}\right) x \rightarrow((x \rightarrow y) \rightsquigarrow y)=1$ and $x \rightsquigarrow((x \rightsquigarrow y) \rightarrow y)=1$;

$\left(\mathrm{psBCI}_{3}\right) x \rightarrow x=x \rightsquigarrow x=1$;

$\left(\mathrm{psBCI}_{4}\right) x \rightarrow y=y \rightsquigarrow x=1 \Rightarrow x=y$;

$\left(\operatorname{psBCI}_{5}\right) x \preceq y$ if and only if $x \rightarrow y=1$ if and only if $x \rightsquigarrow y=1$.

A pseudo-BCK algebra [20] is a pseudo-BCI algebra $\mathfrak{X}=(X ; \rightarrow, \rightsquigarrow, 1)$ satisfying the condition (psBCK), for all $x \in X$ :

$(\mathrm{psBCK}) x \rightarrow 1=1$.

I. Chajda et al. proved that for every pseudo-BCI algebra $x \rightarrow y=1$ if and only if $x \rightsquigarrow y=1$ (see [4, Lemma 2.1]).

Remark 2.1. If $\mathfrak{X}=(X ; \rightarrow, \rightsquigarrow, 1)$ is a pseudo-BCI algebra satisfying $x \rightarrow y=x \rightsquigarrow y$, for all $x, y \in X$, then $\mathfrak{X}=(X ; \rightarrow, 1)$ is a BCI-algebra. Hence, every BCI-algebra is a pseudo-BCI algebra in a natural way.

Remark 2.2. By definition $\left(\mathrm{psBCI}_{1}\right)-\left(\mathrm{psBCI}_{5}\right)$, pseudo-BCK algebras are contained in the class of pseudo-BCI algebras. A pseudo-BCI algebra which is not a pseudo-BCK algebra will be called proper.

From now on, $\mathfrak{X}$ is a pseudo-BCK algebra, unless it is stated.

Proposition 2.1 ([12,17]). In any pseudo-BCK algebra $\mathfrak{X}$ the following conditions hold for all $x, y, z \in X$ :

(1) $x \preceq y$ implies $z \rightarrow x \preceq z \rightarrow y$ and $z \rightsquigarrow x \preceq z \rightsquigarrow y$;

(2) $x \preceq y$ implies $y \rightarrow z \preceq x \rightarrow z$ and $y \rightsquigarrow z \preceq x \rightsquigarrow z$;

(3) $x \rightarrow y \preceq(z \rightarrow x) \rightsquigarrow(z \rightarrow y)$ and $x \rightsquigarrow y \preceq(z \rightsquigarrow x) \rightarrow(z \rightsquigarrow y)$;

(4) $x \rightarrow(y \rightsquigarrow z)=y \rightsquigarrow(x \rightarrow z)$ and $x \rightsquigarrow(y \rightarrow z)=y \rightarrow(x \rightsquigarrow z)$;

(5) $x \preceq y \rightarrow x$ and $x \preceq y \rightsquigarrow x$;

(6) $((x \rightarrow y) \rightsquigarrow y) \rightarrow y=x \rightarrow y$ and $((x \rightsquigarrow y) \rightarrow y) \rightsquigarrow y=x \rightsquigarrow y$.

Theorem 2.1 ([6]). Let $\mathfrak{X}$ be a pseudo-BCK algebra. The following statements are equivalent for all $x, y \in X$ :

(1) $\mathfrak{X}$ is commutative;

(2) $x \rightarrow y=((y \rightarrow x) \rightsquigarrow x) \rightarrow y$ and $x \rightsquigarrow y=((y \rightsquigarrow x) \rightarrow x) \rightsquigarrow y$;

(3) $(x \rightarrow y) \rightsquigarrow y=(((x \rightarrow y) \rightsquigarrow y) \rightarrow x) \rightsquigarrow x$ and $(x \rightsquigarrow y) \rightarrow y=(((x \rightsquigarrow y) \rightarrow y) \rightsquigarrow x) \rightarrow x$;

(4) $x \preceq y$ implies $y=(y \rightarrow x) \rightsquigarrow x=(y \rightsquigarrow x) \rightarrow x$. 
Definition 2.2 ([16]). If there is an element 0 of a pseudo-BCK algebra $\mathfrak{X}$, such that $0 \preceq x$ (i.e., $0 \rightarrow x=0 \rightsquigarrow x=1$ ), for all $x \in X$, then 0 is called the zero of $\mathfrak{X}$. A pseudo-BCK algebra with zero is called bounded pseudo-BCK algebra and it is denoted by $\mathfrak{X}=(X ; \rightarrow, \rightsquigarrow, 0,1)$.

Definition 2.3 ([16]). A pseudo-BCK $(p P)$ algebra is a pseudo-BCK algebra $\mathfrak{X}$ satisfying $(\mathrm{pP})$ condition:

(pP) There exists $x \odot y=\min \{z: x \preceq y \rightarrow z\}=\min \{z: y \preceq x \rightsquigarrow z\}$ for all $x, y \in X$.

Definition $2.4([16,20])$.

(1) A pseudo-BCK lattice is a pseudo-BCK algebra $\mathfrak{X}$ such that $(X ; \preceq)$ is a lattice.

(2) A pseudo-BCK join-semilattice is a pseudo-BCK algebra $\mathfrak{X}$ such that $(X ; \vee)$ is a join-semilattice, and $x \rightarrow y=1$ if and only if $x \vee y=y$.

(3) A pseudo-BCK meet-semilattice is a pseudo-BCK algebra $\mathfrak{X}$ such that $(X ; \wedge)$ is a meet-semilattice, and $x \rightarrow y=1$ if and only if $x \wedge y=x$.

Definition $2.5([16])$. A pseudo-BCK algebra $(p D N)$ is a bounded pseudo-BCK algebra $\mathfrak{X}=(X ; \preceq, \rightarrow, \rightsquigarrow, 0,1)$ satisfying the condition:

$(\mathrm{pDN})\left(x^{\rightarrow}\right)^{\rightsquigarrow}=\left(x^{\rightsquigarrow}\right) \rightarrow=x$, where $x^{\rightarrow}=x \rightarrow 0$ and $x^{\rightsquigarrow}=x \rightsquigarrow 0$ for all $x \in X$.

Definition 2.6 ([12]). A pseudo-BCK algebra $\mathfrak{X}$ is called commutative if for all $x, y, z \in X$, it satisfies the following identities:

$\left(\mathrm{C}_{1}\right)(x \rightarrow y) \rightsquigarrow y=(y \rightarrow x) \rightsquigarrow x ;$

$\left(\mathrm{C}_{2}\right)(x \rightsquigarrow y) \rightarrow y=(y \rightsquigarrow x) \rightarrow x$.

Proposition 2.2 ([6]). Any commutative pseudo-BCK algebra is a join-semilattice with respect to $\preceq$.

Theorem $2.2([8])$. Let $\mathfrak{X}$ be a pseudo-BCK(pDN) algebra. The following statements are equivalent:

(1) $(X ; \preceq)$ is a meet-semilattice;

(2) $(X ; \preceq)$ is a join-semilattice;

(3) $(X ; \preceq)$ is a lattice.

Definition $2.7([6])$. A subset $D$ of a pseudo-BCK algebra $\mathfrak{X}$ is called a deductive system of $\mathfrak{X}$ if it satisfies the following conditions:

$\left(\mathrm{DS}_{1}\right) 1 \in D$;

$\left(\mathrm{DS}_{2}\right) x \in D$ and $x \rightarrow y \in D$ imply $y \in D$.

A subset $D$ of $\mathfrak{X}$ is a deductive system if and only if it satisfies (DS 1 ) and the condition:

$\left(\mathrm{DS}_{3}\right) x \in D$ and $x \rightsquigarrow y \in D$ imply $y \in D$.

We will denote by $\mathfrak{D} S(X)$ the set of all deductive systems of $\mathfrak{X}$.

Definition $2.8([6])$. A deductive system $D$ of a pseudo-BCK algebra $\mathfrak{X}$ is called commutative if it satisfies the following conditions for all $x, y \in X$ : 
$\left(\mathrm{CDS}_{1}\right) y \rightarrow x \in D$ implies $((x \rightarrow y) \rightsquigarrow y) \rightarrow x \in D$;

$\left(\mathrm{CDS}_{2}\right) y \rightsquigarrow x \in D$ implies $((x \rightsquigarrow y) \rightarrow y) \rightsquigarrow x \in D$.

We will denote by $\mathfrak{D} S_{c}(X)$ the set of all commutative deductive systems of a pseudo-BCK algebra $\mathfrak{X}$.

Definition $2.9([3])$. A directoid is a groupoid $\mathfrak{G}=(G ; \vee)$ satisfying the following identities for all $x, y, z \in G$ :

$\left(\mathrm{D}_{1}\right) x \vee x=x$;

$\left(\mathrm{D}_{2}\right)(x \vee y) \vee x=x \vee y$;

$\left(\mathrm{D}_{3}\right) y \vee(x \vee y)=x \vee y$;

$\left(\mathrm{D}_{4}\right) x \vee((x \vee y) \vee z)=(x \vee y) \vee z$.

The relation $\leq$ given by $x \leq y$ if and only if $x \vee y=y$ is a partial order. The binary operation $\vee$ assigns to a pair $\{x, y\}$ is a common upper bound of them.

\section{Pseudo-BCK Algebras Derived from Directoids}

Following the idea used by I. Chajda and J. Kühr [3] for BCK-algebras in what follows we give a generalization of this results for pseudo-BCK algebras. In this section, we assign a semilattice-like structure the sections of which have certain antitone mappings, and also conversely. We have the following results.

Let $\mathfrak{X}$ be a pseudo-BCK algebra. Define binary operations $\vee_{1}$ and $\vee_{2}$ by:

(A) $x \vee_{1} y:=(x \rightarrow y) \rightsquigarrow y$ and $x \vee_{2} y:=(x \rightsquigarrow y) \rightarrow y$ for all $x, y \in X$.

The following examples shows that these operations $\vee_{1}$ and $\vee_{2}$ need not coincide in general.

Example 3.1 ([8]). Consider the set $X=\{0, a, b, c, 1\}$, where $0<a, b<c<1, a, b$ inomparable and the operations $\rightarrow$ and $\rightsquigarrow$ given by the following tables:

\begin{tabular}{c|cccccc|ccccc}
$\rightarrow$ & 0 & $a$ & $b$ & $c$ & 1 & $\rightsquigarrow$ & 0 & $a$ & $b$ & $c$ & 1 \\
\hline 0 & 1 & 1 & 1 & 1 & 1 & 0 & 1 & 1 & 1 & 1 & 1 \\
$a$ & 0 & 1 & $b$ & 1 & 1 & $a$ & $b$ & 1 & $b$ & 1 & 1 \\
$b$ & $a$ & $a$ & 1 & 1 & 1 & $b$ & 0 & $a$ & 1 & 1 & 1 \\
$c$ & 0 & $a$ & $b$ & 1 & 1 & $c$ & 0 & $a$ & $b$ & 1 & 1 \\
1 & 0 & $a$ & $b$ & $c$ & 1 & 1 & 0 & $a$ & $b$ & $c$ & 1
\end{tabular}.

Then $\mathfrak{X}=(X ; \rightarrow, \rightsquigarrow, 0,1)$ is a bounded pseudo-BCK algebra, but $a \vee_{1} 0=(a \rightarrow 0) \rightsquigarrow 0=0 \rightsquigarrow 0=1 \neq a \vee_{2} 0=(a \rightsquigarrow 0) \rightarrow 0=b \rightarrow 0=a$.

Theorem 3.1. Let $\mathfrak{X}$ be a pseudo-BCK algebra. For every $a \in X$, define unary operations $\rightarrow a$ and ${ }^{\rightsquigarrow a}$ by $x^{\rightarrow a}=x \rightarrow a$ and $x^{\rightsquigarrow a}=x \rightsquigarrow a$. Then the algebraic structure $\mathfrak{S}(\mathfrak{X})=\left(X ; \vee_{1}, \vee_{2},\left({ }^{\rightarrow a}\right)_{a \in X},\left({ }^{\rightsquigarrow a}\right)_{a \in X}, 1\right)$ satisfies the following quasi-identities:

(1) $x \vee_{1} 1=1$ and $x \vee_{2} 1=1$;

(2) $x^{\rightarrow x}=1$ and $x^{\rightsquigarrow x}=1$;

(3) $1^{\rightarrow x}=x$ and $1^{\rightsquigarrow x}=x$;

(4) $x \vee_{1} x=x$ and $x \vee_{2} x=x$; 
(5) $\left(x \vee_{1} y\right)^{\rightarrow y}=x \rightarrow y$ and $\left(x \vee_{2} y\right)^{\rightsquigarrow y}=x \rightsquigarrow y$;

(6) $x \preceq y$ if and only if $x \vee_{1} y=y$ and $x \vee_{2} y=y$;

(7) $x \vee_{1} y=y$ and $y \vee_{1} x=x$ imply $x=y$ and $x \vee_{2} y=y$ and $y \vee_{2} x=x$ imply $x=y$;

(8) $x \vee_{1} y=\left(x \vee_{1} y\right) \vee_{1} y=x \vee_{1}\left(x \vee_{1} y\right)=y \vee_{1}\left(x \vee_{1} y\right)$ and $x \vee_{2} y=\left(x \vee_{2} y\right) \vee_{2} y=x \vee_{2}\left(x \vee_{2} y\right)=y \vee_{2}\left(x \vee_{2} y\right)$

(9) $\left(x \vee_{1} z\right) \vee_{1}\left(\left(x \vee_{1} y\right) \vee_{1} z\right)=\left(x \vee_{1} y\right) \vee_{1} z$ and $\left(x \vee_{2} z\right) \vee_{2}\left(\left(x \vee_{2} y\right) \vee_{2} z\right)=\left(x \vee_{2} y\right) \vee_{2} z$

(10) $x \vee_{1} y=\left(x \vee_{1} y\right)^{\rightarrow y \rightsquigarrow y}=\left(\left(x \vee_{1} y\right)^{\rightarrow y} \vee_{2} y\right)^{\rightsquigarrow y}$ and $x \vee_{2} y=\left(x \vee_{2} y\right)^{\rightsquigarrow y \rightarrow y}=\left(\left(x \vee_{2} y\right)^{\rightsquigarrow y} \vee_{1} y\right)^{\rightarrow y}$

(11) $\left(x \vee_{1}\left(y \vee_{2} z\right)^{\rightsquigarrow z}\right)^{\rightarrow\left(y \vee_{2} z\right)^{\rightsquigarrow z}}=\left(y \vee_{2}\left(x \vee_{1} z\right)^{\rightarrow z}\right)^{\rightsquigarrow\left(x \vee_{1} z\right)^{\rightarrow z}}$;

(12) $\left(x \vee_{1} y\right)^{\rightarrow y} \vee_{2}\left(\left(x \vee_{1} z\right) \vee_{1}\left(y \vee_{1} z\right)\right) \rightarrow\left(y \vee_{1} z\right)=\left(\left(x \vee_{1} z\right) \vee_{1}\left(y \vee_{1} z\right)\right) \rightarrow\left(y \vee_{1} z\right)$ and $\left(x \vee_{2} y\right)^{\rightsquigarrow y} \vee_{1}\left(\left(x \vee_{2} z\right) \vee_{2}\left(y \vee_{2} z\right)\right)^{\rightsquigarrow\left(y \vee_{2} z\right)}=\left(\left(x \vee_{2} z\right) \vee_{2}\left(y \vee_{2} z\right)\right)^{\rightsquigarrow\left(y \vee_{2} z\right)}$;

(13) $\left(\left(x \vee_{2} z\right)^{\rightsquigarrow z} \vee_{1}\left(y \vee_{1} z\right)\right) \rightarrow\left(y \vee_{1} z\right)=\left(\left(y \vee_{1} z\right)^{\rightarrow z} \vee_{2}\left(x \vee_{2} z\right)\right)^{\rightsquigarrow\left(x \vee_{2} z\right)}$ and $\left(\left(x \vee_{1} z\right)^{\rightarrow z} \vee_{2}\left(y \vee_{2} z\right)\right)^{\rightsquigarrow\left(y \vee_{2} z\right)}=\left(\left(y \vee_{2} z\right)^{\rightsquigarrow z} \vee_{1}\left(x \vee_{1} z\right)\right) \rightarrow\left(x \vee_{1} z\right)$;

(14) $\left(\left(x \vee_{1} y\right) \vee_{1} x\right)^{\rightarrow x}=\left(x \vee_{1} y\right)^{\rightarrow x}$ and $\left(\left(x \vee_{2} y\right) \vee_{2} x\right)^{\rightsquigarrow x}=\left(x \vee_{2} y\right)^{\rightsquigarrow x}$.

Proof. The proof of (1)-(6) is straightforward by the definition and properties of pseudo-BCK algebras. (6) Assume that $x \preceq y$. Then $x \vee_{1} y=(x \rightarrow y) \rightsquigarrow y=1 \rightsquigarrow$ $y=y$. Also,

$$
x \vee_{2} y=(x \rightsquigarrow y) \rightarrow y=1 \rightarrow y=y .
$$

Conversely, suppose that $x \vee_{1} y=y$ and $x \vee_{2} y=y$. Since

$$
x \rightarrow y=x \rightarrow((x \rightarrow y) \rightsquigarrow y)=(x \rightarrow y) \rightsquigarrow(x \rightarrow y)=1
$$

and

$$
x \rightsquigarrow y=x \rightsquigarrow((x \rightsquigarrow y) \rightarrow y)=(x \rightsquigarrow y) \rightarrow(x \rightsquigarrow y)=1,
$$

then $x \preceq y$.

(7) Suppose that $(x \rightarrow y) \rightsquigarrow y=y$ and $(y \rightarrow x) \rightsquigarrow x=x$. Then by $\left(\mathrm{psBCI}_{3}\right)$ we have

$$
x \rightarrow y=x \rightarrow((x \rightarrow y) \rightsquigarrow y)=(x \rightarrow y) \rightsquigarrow(x \rightarrow y)=1
$$

and

$$
y \rightsquigarrow x=y \rightsquigarrow((y \rightsquigarrow x) \rightarrow x)=(y \rightsquigarrow x) \rightarrow(y \rightsquigarrow x)=1 .
$$

Now, using $\left(\mathrm{psBCI}_{5}\right) x=y$. By a similar argument the second part is valid.

(8) By Proposition 2.1 (6), we have

$$
\begin{aligned}
\left(x \vee_{1} y\right) \vee_{1} y & =\left(\left(x \vee_{1} y\right) \rightarrow y\right) \rightsquigarrow y \\
& =(((x \rightarrow y) \rightsquigarrow y) \rightarrow y) \rightsquigarrow y \\
& =(x \rightarrow y) \rightsquigarrow y \\
& =x \vee_{1} y .
\end{aligned}
$$

Similarly, we see that $x \vee_{1}\left(x \vee_{1} y\right)=x \vee_{1} y$ and $y \vee_{1}\left(x \vee_{1} y\right)=x \vee_{1} y$. 
(9) According to ( $\left.\mathrm{psBCI}_{2}\right), x \preceq x \vee_{1} y$. By Proposition 2.1 (1) and (2), we have $x \vee_{1} z \preceq\left(x \vee_{1} y\right) \vee_{1} z$. Now, using (6) it follows that $\left(x \vee_{1} z\right) \vee_{1}\left(\left(x \vee_{1} y\right) \vee_{1} z\right)=\left(x \vee_{1} y\right) \vee_{1} z$. By a similar argument we can verify $x \vee_{2} y=\left(x \vee_{2} y\right) \vee_{2} y=x \vee_{2}\left(x \vee_{2} y\right)=y \vee_{2}\left(x \vee_{2} y\right)$.

(10) $\left(x \vee_{1} y\right)^{\rightarrow y \rightsquigarrow y}=\left(\left(x \vee_{1} y\right) \rightarrow y\right) \rightsquigarrow y=x \vee_{1} y$ and $\left(x \vee_{2} y\right)^{\rightsquigarrow y \rightarrow y}=\left(\left(x \vee_{2} y\right) \rightsquigarrow\right.$ $y) \rightarrow y=x \vee_{2} y$. By Proposition 2.1 (6), we have

$$
\begin{aligned}
\left(\left(x \vee_{1} y\right)^{\rightarrow y} \vee_{2} y\right)^{\rightsquigarrow y} & =\left(\left(\left(x \vee_{1} y\right) \rightarrow y\right) \vee_{2} y\right)^{\rightsquigarrow y} \\
& =\left((((x \rightarrow y) \rightsquigarrow y) \rightarrow y) \vee_{2} y\right)^{\rightsquigarrow y} \\
& =\left((x \rightarrow y) \vee_{2} y\right)^{\rightsquigarrow y} \\
& =(((x \rightarrow y) \rightsquigarrow y) \rightarrow y)^{\rightsquigarrow y} \\
& =(x \rightarrow y)^{\rightsquigarrow y} \\
& =(x \rightarrow y) \rightsquigarrow y \\
& =x \vee_{1} y .
\end{aligned}
$$

Also, the proof of the second part is similar.

(11) From (5) and Proposition 2.1 (4), we conclude

$$
\begin{aligned}
\left(x \vee_{1}\left(y \vee_{2} z\right)^{\rightsquigarrow z}\right) \rightarrow\left(y \vee_{2} z\right)^{\rightsquigarrow z} & =\left(x \vee_{1}(y \rightsquigarrow z)\right)^{\rightarrow(y \rightsquigarrow z)} \\
& =x \rightarrow(y \rightsquigarrow z)=y \rightsquigarrow(x \rightarrow z) \\
& =\left(y \vee_{2}(x \rightarrow z)\right)^{\rightsquigarrow(x \rightarrow z)} \\
& =\left(y \vee_{2}\left(x \vee_{1} z\right)^{\rightarrow z}\right)^{\rightsquigarrow\left(x \vee_{1} z\right)^{\rightarrow z}} .
\end{aligned}
$$

(12) Using (5), we have

$$
\begin{aligned}
\left(\left(x \vee_{1} z\right) \vee_{1}\left(y \vee_{1} z\right)\right) \rightarrow\left(y \vee_{1} z\right) & =\left(x \vee_{1} z\right) \rightarrow\left(y \vee_{1} z\right) \\
& =((x \rightarrow z) \rightsquigarrow z) \rightarrow((y \rightarrow z) \rightsquigarrow z) \\
& =(y \rightarrow z) \rightsquigarrow(((x \rightarrow z) \rightsquigarrow z) \rightarrow z) \\
& =(y \rightarrow z) \rightsquigarrow(x \rightarrow z) .
\end{aligned}
$$

We have $(x \rightarrow y) \vee_{2}((y \rightarrow z) \rightsquigarrow(x \rightarrow z))=1 \rightarrow((y \rightarrow z) \rightsquigarrow(x \rightarrow z))$. From this and $\left(\mathrm{psBCI}_{1}\right)$ we conclude

$$
\begin{aligned}
\left(x \vee_{1} y\right)^{\rightarrow y} \vee_{2}\left(\left(x \vee_{1} z\right) \vee_{1}\left(y \vee_{1} z\right)\right)^{\rightarrow\left(y \vee_{1} z\right)} & =\left(x \vee_{1} y\right)^{\rightarrow y} \vee_{2}((y \rightarrow z) \rightsquigarrow(x \rightarrow z)) \\
& =(x \rightarrow y) \vee_{2}((y \rightarrow z) \rightsquigarrow(x \rightarrow z)) \\
& =1 \rightarrow((y \rightarrow z) \rightsquigarrow(x \rightarrow z)) \\
& =(y \rightarrow z) \rightsquigarrow(x \rightarrow z) \\
& =\left(\left(x \vee_{1} z\right) \vee_{1}\left(y \vee_{1} z\right)\right) \rightarrow\left(y \vee_{1} z\right)
\end{aligned}
$$


(13) Applying (5), we have

$$
\begin{aligned}
\left(\left(x \vee_{2} z\right)^{\rightsquigarrow z} \vee_{1}\left(y \vee_{1} z\right)\right)^{\rightarrow\left(y \vee_{1} z\right)} & =(x \rightsquigarrow z) \rightarrow\left(y \vee_{1} z\right) \\
& =(x \rightsquigarrow z) \rightarrow((y \rightarrow z) \rightsquigarrow z) \\
& =(y \rightarrow z) \rightsquigarrow((x \rightsquigarrow z) \rightarrow z) \\
& =(y \rightarrow z) \rightsquigarrow\left(x \vee_{2} z\right) \\
& =\left(\left(y \vee_{1} z\right)^{\rightarrow z} \vee_{2}\left(x \vee_{2} z\right)\right)^{\rightsquigarrow\left(x \vee_{2} z\right)} .
\end{aligned}
$$

By a similar argument we have

$$
\left(\left(x \vee_{1} z\right)^{\rightarrow z} \vee_{2}\left(y \vee_{2} z\right)\right)^{\rightsquigarrow\left(y \vee_{2} z\right)}=\left(\left(y \vee_{2} z\right)^{\rightsquigarrow z} \vee_{1}\left(x \vee_{1} z\right)\right)^{\rightarrow\left(x \vee_{1} z\right)} \text {. }
$$

(14) Using Proposition 2.1 (6), we get

$$
\begin{aligned}
\left(\left(x \vee_{1} y\right) \vee_{1} x\right)^{\rightarrow x} & =\left(((x \rightarrow y) \rightsquigarrow y) \vee_{1} x\right)^{\rightarrow x} \\
& =((((x \rightarrow y) \rightsquigarrow y) \rightarrow x) \rightsquigarrow x)^{\rightarrow x} \\
& =((x \rightarrow y) \rightsquigarrow y)^{\rightarrow x} \\
& =((x \rightarrow y) \rightsquigarrow y) \rightarrow x \\
& =\left(x \vee_{1} y\right) \rightarrow x \\
& =\left(x \vee_{1} y\right)^{\rightarrow x}
\end{aligned}
$$

Similarly, $\left(\left(x \vee_{2} y\right) \vee_{2} x\right)^{\rightsquigarrow x}=\left(x \vee_{2} y\right)^{\rightsquigarrow x}$.

Lemma 3.1. Let $\mathfrak{X}=\left(X ; \vee_{1}, \vee_{2}\right)$ be an algebra of type $(2,2)$ satisfying the quasiidentities (4), (7), (8) and (9) of Theorem 3.1. Then the binary relation $\preceq$ defined by

(B) $x \preceq y$ if and only if $x \vee_{1} y=y$ and $x \vee_{2} y=y$ is a partial order on $X$.

Proof. By (4) and (7), $\preceq$ is reflexive and antisymmetric. For transitivity, assume that $x \preceq y$ and $y \preceq z$. Using (8) and (9), we get

$$
\begin{aligned}
x \vee_{1} z & =\left(x \vee_{1} z\right) \vee_{1} z \\
& =\left(x \vee_{1} z\right) \vee_{1}\left(y \vee_{1} z\right) \\
& =\left(x \vee_{1} z\right) \vee_{1}\left(\left(x \vee_{1} y\right) \vee_{1} z\right) \\
& =\left(x \vee_{1} y\right) \vee_{1} z \\
& =y \vee_{1} z=z
\end{aligned}
$$

and if $x \vee_{2} y=y$ and $y \vee_{2} z=z$, then we have

$$
\begin{aligned}
x \vee_{2} z & =\left(x \vee_{2} z\right) \vee_{2} z \\
& =\left(x \vee_{2} z\right) \vee_{2}\left(y \vee_{2} z\right) \\
& =\left(x \vee_{2} z\right) \vee_{2}\left(\left(x \vee_{2} y\right) \vee_{2} z\right) \\
& =\left(x \vee_{2} y\right) \vee_{2} z \\
& =y \vee_{2} z=z .
\end{aligned}
$$


Thus, $\preceq$ is a partial order on $X$.

The following example shows that for every pseudo-BCK algebra $\mathfrak{X},\left(X ; \vee_{1}\right)$ and $\left(X ; \vee_{2}\right)$ are not directoids in general.

Example 3.2. Let $\mathfrak{X}$ be the algebra given in Example 3.1. Then $\left(X ; \vee_{1}\right)$ and $\left(X ; \vee_{2}\right)$ are not directoids, since

$c \vee_{1} 0=(c \rightarrow 0) \rightsquigarrow 0=0 \rightsquigarrow 0=1 \neq\left(c \vee_{1} 0\right) \vee_{1} c=0 \vee_{1} c=(0 \rightarrow c) \rightsquigarrow c=1 \rightsquigarrow c=c$ and

$c \vee_{2} 0=(c \rightsquigarrow 0) \rightarrow 0=0 \rightarrow 0=1 \neq\left(c \vee_{2} 0\right) \vee_{2} c=0 \vee_{2} c=(0 \rightsquigarrow c) \rightarrow c=1 \rightarrow c=c$.

Theorem 3.2. Let $\mathfrak{X}$ be a pseudo-BCK algebra, $\vee_{1}$ and $\vee_{2}$ be the binary operations defined by $(\mathrm{A})$. Then the following conditions are equivalent:

(1) $\left(X ; \vee_{1}\right)$ and $\left(X ; \vee_{2}\right)$ are directoids;

(2) $\mathfrak{X}$ is a commutative pseudo- $B C K$ algebra;

(3) $(X ; \preceq)$ is a join-semilattice, where $\preceq$ is defined by (B).

Proof. $(1) \Rightarrow(2)$ Assume that $\left(X ; \vee_{1}\right)$ is a directoid. Then $x \preceq y$ implies $y \vee_{1} x=y$ and so $\mathfrak{X}$ satisfies the quasi-identity

$$
x \preceq y \Rightarrow y=(y \rightarrow x) \rightsquigarrow x .
$$

Similary, $x \preceq y$ implies $y=(y \rightsquigarrow x) \rightarrow x$. Therefore, $\mathfrak{X}$ is a commutative pseudo-BCK algebra by Theorem 2.1 .

$(2) \Rightarrow(3)$ It follows from Proposition 2.2 .

$(3) \Rightarrow(1)$ It is obvious that every join-semilattice is a directoid.

Corollary 3.1. Let $\mathfrak{X}$ be a pseudo-BCK $(p D N), \vee_{1}$ and $\vee_{2}$ be the binary operations defined by $(\mathrm{A})$. Then the following conditions are equivalent:

(1) $\left(X ; \vee_{1}\right)$ and $\left(X ; \vee_{2}\right)$ are directoids;

(2) $\mathfrak{X}$ is a commutative pseudo-BCK algebra;

(3) $(X ; \preceq)$ is a join-semilattice;

(4) $(X ; \preceq)$ is a meet-semilattice;

(5) $(X ; \preceq)$ is a lattice.

Proof. It follows from Theorems 3.2 and 2.2.

Corollary 3.2. Let $\mathfrak{X}$ be a pseudo- $B C K(p D N), \vee_{1}$ and $\vee_{2}$ be the binary operations defined by $(\mathrm{A})$. Then the following conditions are equivalent:

(1) $\left(X ; \vee_{1}\right)$ and $\left(X ; \vee_{2}\right)$ are directoids;

(2) $\mathfrak{X}$ is a commutative pseudo-BCK algebra;

(3) $\{1\} \in \mathfrak{D} S_{c}(X)$;

(4) $\mathfrak{D} S(X)=\mathfrak{D} S_{c}(X)$.

Proof. It follows from Theorem 3.2 and [6, Corollary 4.6, Theorem 4.7 and Corollary 4.8]. 
In [8], L. C. Ciungu proved that for every pseudo-BCK(pDN) lattice the following conditions are equivalent (see [8, Proposition 3.5]):

$\left(\mathrm{P}_{1}\right)(x \wedge y) \rightarrow z=(x \rightarrow z) \vee(y \rightarrow z)$ and $(x \wedge y) \rightsquigarrow z=(x \rightsquigarrow z) \vee(y \rightsquigarrow z)$;

$\left(\mathrm{P}_{2}\right) z \rightarrow(x \vee y)=(z \rightarrow x) \vee(z \rightarrow y)$ and $z \rightsquigarrow(x \vee y)=(z \rightsquigarrow x) \vee(z \rightsquigarrow y)$

Also, she showed that the class of pseudo-BCK $(\mathrm{pDN})$ lattices satisfies the conditions $\left(\mathrm{P}_{1}\right)$ and $\left(\mathrm{P}_{2}\right)$ is not empty, since every pseudo-MV algebra satisfies these conditions. Further, It was proved that if a pseudo-BCK $(\mathrm{pDN})$ lattice $\mathfrak{X}$ satisfying $\left(\mathrm{P}_{1}\right)$ or $\left(\mathrm{P}_{2}\right)$, then $(X ; \preceq)$ is a distributive lattice (see $[8$, Theorem 3.4 , Corollary 3.2]).

Theorem 3.3. Let $\mathfrak{S}=\left(S ; \vee_{1}, \vee_{2},\left({ }^{\rightarrow a}\right)_{a \in S},\left({ }^{\sim a}\right)_{a \in S}, 1\right)$ be a structure algebraic, where $\vee_{1}$ and $\vee_{2}$ are binary operations on $S$ and for each $a \in S, \rightarrow a$ and ${ }^{\rightsquigarrow a}$ are unary operations on $\left\{x \in S: a \vee_{1} x=1\right.$ and $\left.a \vee_{2} x=1\right\}$ and 1 is a distinguished element of $S$, satisfying the quasi-identities (1)-(12) from Theorem 3.1. Define the new binary operations $\rightarrow$ and $\rightsquigarrow$ on $S$ by

(C) $x \rightarrow y=\left(x \vee_{1} y\right)^{\rightarrow y}$ and $x \rightsquigarrow y=\left(x \vee_{2} y\right)^{\rightsquigarrow y}$.

Then $\mathfrak{X}(\mathfrak{S})=(S ; \rightarrow, \rightsquigarrow, 1)$ is a pseudo-BCK algebra.

Proof. The definition of $\rightarrow$ and $\rightsquigarrow$ are well define from Theorem 3.1 (8). Furthermore, we note that

(D) $x \vee_{1} y=y$ and $x \vee_{2} y=y$ if and only if $x \rightarrow y=1$ and $x \rightsquigarrow y=1$.

Indeed, if $x \vee_{1} y=y$, then $x \rightarrow y=\left(x \vee_{1} y\right)^{\rightarrow y}=y^{\rightarrow y}=1$, by Theorem 3.1 (2). Similarly, if $x \vee_{2} y=y$, then $x \rightsquigarrow y=\left(x \vee_{2} y\right)^{\rightsquigarrow y}=y^{\rightsquigarrow y}=1$.

For conversely, $1=x \rightarrow y=\left(x \vee_{1} y\right)^{\rightarrow y}$ implies $y=1^{\rightsquigarrow y}=\left(x \vee_{1} y\right)^{\rightarrow y \rightsquigarrow y}=x \vee_{1} y$. Also, $1=x \rightsquigarrow y=\left(x \vee_{2} y\right)^{\rightsquigarrow y}$ implies $y=1^{\rightarrow y}=\left(x \vee_{2} y\right)^{\rightsquigarrow y \rightarrow y}=x \vee_{2} y$, by Theorem 3.1 (3) and (10). Now, we verify the axioms of pseudo-BCK algebras as follows.

(psBCI $\left.{ }_{1}\right)$ Using Theorem 3.1 (5) and (12), we obtain

$$
\begin{aligned}
(x \rightarrow y) \vee_{2}\left(\left(x \vee_{1} z\right) \rightarrow\left(y \vee_{1} z\right)\right) & =\left(x \vee_{1} y\right)^{\rightarrow y} \vee_{2}\left(\left(x \vee_{1} z\right) \vee_{1}\left(y \vee_{1} z\right)\right) \rightarrow\left(y \vee_{1} z\right) \\
& =\left(\left(x \vee_{1} z\right) \vee_{1}\left(y \vee_{1} z\right)\right) \rightarrow\left(y \vee_{1} z\right) \\
& =\left(x \vee_{1} z\right) \rightarrow\left(y \vee_{1} z\right)
\end{aligned}
$$

Thus, $(x \rightarrow y) \rightsquigarrow\left(\left(x \vee_{1} z\right) \rightarrow\left(y \vee_{1} z\right)\right)=1$.

Also, according to Theorem 3.1 (10) and (11), we get

$$
(x \rightarrow y) \rightsquigarrow y=\left(\left(x \vee_{1} y\right)^{\rightarrow y} \vee_{2} y\right)^{\rightsquigarrow y}=x \vee_{1} y
$$

and

$$
x \rightarrow(y \rightsquigarrow z)=\left(x \vee_{1}\left(y \vee_{2} z\right)^{\rightsquigarrow z}\right)^{\rightarrow\left(y \vee_{2} z\right)}=\left(y \vee_{2}\left(x \vee_{1} z\right)^{\rightarrow z}\right)^{\rightsquigarrow\left(x \vee_{1} z\right)}=y \rightsquigarrow(x \rightarrow z)
$$

Then

$$
\begin{aligned}
\left(x \vee_{1} z\right) \rightarrow\left(y \vee_{1} z\right) & =((x \rightarrow z) \rightsquigarrow z) \rightarrow((y \rightarrow z) \rightsquigarrow z) \\
& =(y \rightarrow z) \rightsquigarrow(((x \rightarrow z) \rightsquigarrow z) \rightarrow z) \\
& =(y \rightarrow z) \rightsquigarrow(x \rightarrow z),
\end{aligned}
$$


since

$$
\begin{aligned}
((x \rightarrow z) \rightarrow z) \rightsquigarrow z & =\left(\left(\left(x \vee_{1} z\right)^{\rightarrow z} \vee_{2} z\right)^{\rightarrow z} \vee_{1} z\right)^{\rightarrow z} \\
& =\left(\left(x \vee_{1} z\right) \vee_{1} z\right)^{\rightarrow z} \\
& =\left(x \vee_{1} z\right)^{\rightarrow z} \\
& =x \rightarrow z
\end{aligned}
$$

Altogether, we have

$$
(x \rightarrow y) \rightsquigarrow((y \rightarrow z) \rightsquigarrow(x \rightarrow z))=(x \rightarrow y) \rightsquigarrow\left(\left(x \vee_{1} z\right) \rightarrow\left(y \vee_{1} z\right)\right)=1 .
$$

The second part of axiom ( $\left.\mathrm{psBCI}_{1}\right)$ follows by duality.

$\left(\mathrm{psBCI}_{2}\right)$ Using Theorem 3.1 (10), we get

$$
(x \rightarrow y) \rightsquigarrow y=\left(\left(x \vee_{1} y\right)^{\rightarrow y} \vee_{2} y\right)^{\rightsquigarrow y}=x \vee_{1} y .
$$

Hence, $x \vee_{1}((x \rightarrow y) \rightsquigarrow y)=x \vee_{1}\left(x \vee_{1} y\right)=x \vee_{1} y=(x \rightarrow y) \rightsquigarrow y$. Then $x \rightarrow((x \rightarrow y) \rightsquigarrow y)=1$.

By a similar argument we have $x \rightsquigarrow((x \rightsquigarrow y) \rightarrow y)=1$.

( $\left.\operatorname{psBCI}_{3}\right)$ Applying (D), from $x \vee_{1} x=x \vee_{2} x=x$ it follows $x \rightarrow x=x \rightsquigarrow x=1$.

$\left(\mathrm{psBCI}_{4}\right)$ If $x \rightarrow y=1$ and $y \rightarrow x=1$, then by Theorem 3.1 (6), we have $x \vee_{1} y=y$ and $y \vee_{1} x=x$. Now, using Theorem 3.1 (7), it follows $x=y$.

$\left(\mathrm{psBCI}_{5}\right)$ This follows from Theorem 3.1 (6) and (D).

(psBCK) By Theorem 3.1 (1), $x \vee_{1} 1=x \vee_{2} 1=1$. From (D) we see that $x \rightarrow 1=$ $x \rightsquigarrow 1=1$.

Theorem 3.4. Let $\mathfrak{S}=\left(S ; \vee_{1}, \vee_{2},(\rightarrow a)_{a \in S},\left({ }^{\sim a}\right)_{a \in S}, 1\right)$ be an algebra as in Theorem 3.3 satisfying (1)-(12) of Theorem 3.1 and $\mathfrak{X}$ be a pseudo-BCK algebra. Then $\mathfrak{X}(\mathfrak{S}(\mathfrak{X}))=\mathfrak{X}$ and $\mathfrak{S}(\mathfrak{X}(\mathfrak{S}))=\mathfrak{S}$.

Proof. By Theorem 3.1, $\mathfrak{S}(\mathfrak{X})=\left(X ; \vee_{1}, \vee_{2},\left({ }^{\rightarrow a}\right)_{a \in X},\left({ }^{\rightsquigarrow a}\right)_{a \in X}, 1\right)$ is the structure satisfying (1)-(12) which is assigned to a given pseudo-BCK algebra $\mathfrak{X}$. Then in $\mathfrak{X}(\mathfrak{S}(\mathfrak{X}))=\left(X ; \rightarrow_{1}, \rightsquigarrow_{1}, 1\right)$ we have

$$
x \rightarrow_{1} y=\left(x \vee_{1} y\right)^{\rightarrow y}=((x \rightarrow y) \rightsquigarrow y) \rightarrow y=x \rightarrow y
$$

and

$$
x \rightsquigarrow_{2} y=\left(x \vee_{2} y\right)^{\rightsquigarrow y}=((x \rightsquigarrow y) \rightarrow y) \rightsquigarrow y=x \rightsquigarrow y .
$$

Therefore, $\mathfrak{X}(\mathfrak{S}(\mathfrak{X}))=\mathfrak{X}$.

Also, assume that $\mathfrak{S}=\left(S ; \vee_{1}, \vee_{2},\left({ }^{\rightarrow a}\right)_{a \in S},\left({ }^{\cdots a}\right)_{a \in S}, 1\right)$ is a structure that satisfies (1)-(12) of Theorem 3.1, $\mathfrak{X}(\mathfrak{S})=(S ; \rightarrow, \rightsquigarrow, 1)$ its corresponding pseudo-BCK algebra (cf. Theorem 3.3) and $\mathfrak{S}(\mathfrak{X}(\mathfrak{S}))=\left(S ; \sqcup_{1}, \sqcup_{2},\left(r_{1 a}\right)_{a \in S},\left(r_{2 a}\right)_{a \in S}, 1\right)$. Then

$$
x \sqcup_{1} y=(x \rightarrow y) \rightsquigarrow y=\left(\left(x \vee_{1} y\right)^{\rightarrow y} \vee_{2} y\right)^{\rightsquigarrow y}=x \vee_{1} y
$$

and

$$
x \sqcup_{2} y=(x \rightsquigarrow y) \rightarrow y=\left(\left(x \vee_{2} y\right)^{\rightsquigarrow y} \vee_{1} y\right)^{\rightarrow y}=x \vee_{2} y .
$$


Further, for $x \in[a, 1]$, we have

$$
r_{1 a}(x)=x \rightarrow a=\left(x \vee_{1} a\right)^{\rightarrow a}=\left(\left(a \vee_{1} x\right) \vee_{1} a\right)^{\rightarrow a}=\left(a \vee_{1} x\right)^{\rightarrow a}=x^{\rightarrow a}
$$

and

$$
r_{2 a}(x)=x \rightsquigarrow a=\left(x \vee_{2} a\right)^{\rightsquigarrow a}=\left(\left(a \vee_{2} x\right) \vee_{2} a\right)^{\rightsquigarrow a}=\left(a \vee_{2} x\right)^{\rightsquigarrow a}=x^{\rightsquigarrow a} .
$$

Therefore, $\mathfrak{S}(\mathfrak{X}(\mathfrak{S}))=\mathfrak{S}$.

Corollary 3.3. Let $\mathfrak{S}=\left(S ; \vee_{1}, \vee_{2},(\rightarrow a)_{a \in S},\left({ }^{\rightsquigarrow a}\right)_{a \in S}, 1\right)$ be an algebraic structure satisfying (1)-(13) of Theorem 3.1. Then the relation defined by $(B)$ is a partial order on $S, 1$ is the greatest element of $S$ and for every $x, y \in S, x, y \preceq x \vee y$, where $\vee=\vee_{1}=\vee_{2}$. Moreover, for each $a \in S, \rightarrow a$ and ${ }^{\rightsquigarrow a}$ are antitone mappings on $[a, 1]=\{x \in S: a \preceq x\}$.

\section{Conclusion}

We consider that this paper could contribute to the study of algebraic structures and to the development of pseudo-BCK algebras. So, we hope it would be served as a foundation and another topic of research to define and investigate among algebraic structures derived from pseudo-BCK algebras. As another direction of research, one could investigate relationship between commutative pseudo-valuation on pseudo-BCK algebras with directoids.

Acknowledgements. The author would like to express their sincere thanks to the referees for the valuable suggestions which lead to an improvement of this paper. Support of the research by Payame Noor University, the office of Vice Chancellor for Research, Grant No. 49103/7, is gratefully acknowledged.

\section{REFERENCES}

[1] R. A. Borzooei, A. B. Saeid, A. Rezaei, A. Radfar and R. Ameri, On pseudo-BE algebras, Discuss. Math. Gen. Algebra Appl. 33 (2013), 95-108.

[2] R. A. Borzooei, A. B. Saeid, A. Rezaei, A. Radfar and R. Ameri, Distributive pseudo-BE algebras, Fasc. Math. 54 (2015), 21-39.

[3] I. Chajda and J. Kühr, Algebraic structures derived from BCK-algebras, Miskolc Math. Notes 8(1) (2007), 11-21.

[4] I. Chajda and H. Länger, An ordered structure of pseudo-BCI algebras, Math. Bohem. 141(1) (2016), 91-98.

[5] L. C. Ciungu, Commutative pseudo-BE algebras, Iran. J. Fuzzy Syst. 13(1) (2016), 131-144.

[6] L. C. Ciungu, Commutative deductive systems of pseudo BCK-algebras, Soft Computing 22(4) (2018), 1189-1201.

[7] L. C. Ciungu, Non-Commutative Multiple-Valued Logic Algebras, Springer, Cham, Heidelberg, New York, Dordrecht, London, 2014.

[8] L. C. Ciungu, On pseudo-BCK algebras with pseudo-double negation, An. Univ. Craiova Ser. Mat. Inform. 37(1) (2010), 19-26.

[9] W. A. Dudek and Y. B. Jun, Pseudo-BCI algebras, East Asian Math. J. 24 (2008), 187-190.

[10] G. Georgescu and A. Iorgulescu, Pseudo-MV algebras, Multiple-Valued Logic 9 (2001), 95-135. 
[11] G. Georgescu and A. Iorgulescu, Pseudo-BL algebras: a non-commutative extension of BLalgebras, Fifth International Conference FSTA 2000, Slovakia, February, 2000, 90-92.

[12] G. Georgescu and A. Iorgulescu, Pseudo-BCK Algebras: an Extension of BCK-Algebras, DMTCS01: Combinatorics, Computability and Logic, Springer, London, 2001, 97-114.

[13] G. Georgescu, L. Leustean and V. Preoteasa, Pseudo-hoops, J. Mult.-Valued Logic Soft Comput. 11 (2005), 153-184.

[14] R. Halaš and J. Kühr, Deductive systems and annihilators of pseudo-BCK algebras, Italian Journal of Pure and Applied Mathematics 25 (2009), 83-94.

[15] Y. Imai and K. Iséki, On axiom systems of propositional calculi, Proc. Japan Acad. 42 (1966), 19-22.

[16] A. Iorgulescu, Classes of pseudo-BCK algebras - Part I, J. Mult.-Valued Logic Soft Comput. 12 (2006), 71-130.

[17] A. Iorgulescu, Algebras of Logic as BCK-Algebras, Academy of Economic Studies Press, Bucharest, 2008.

[18] A. Iorgulescu, Implicative vs. Groups and Generalizations, Matrix Rom, Bucharest, 2018.

[19] K. Iséki, An algebra related with a propositional calculus, Proc. Japan Acad. 42 (1966), 26-29.

[20] J. Kühr, Pseudo-BCK semilattices, Demonstr. Math. 40 (2007), 495-516.

[21] J. Kühr, Pseudo-BCK Algebras and Related Structures, Univerzita Palackeho v Olomouci, Olomouci, 2007.

[22] J. Kühr, Commutative pseudo-BCK algebras, Southeast Asian Bull. Math. 33 (2009), 451-475.

[23] J. Meng and Y. B. Jun, BCK-Algebras, Kyung Moon Sa Co., Seoul, Korea, 1994.

[24] J. Rachůnek, A non commutative generalization of $M V$-algebras, Czechoslovak Math. J. 52(2) (2002), 255-273.

[25] A. Rezaei, A new trend to pseudo-BCK algebras, in: Proceeding of $6^{\text {th }}$ Iranian Joint Congress on Fuzzy and Intelligent Systems (CFIS), Kerman, Iran, 2018, 117-119.

[26] A. Rezaei, A. B. Saeid, A. Radfar and R. A. Borzooei, Congruence relations on pseudo-BE algebras, An. Univ. Craiova Ser. Mat. Inform. 41 (2014), 166-176.

[27] A. Rezaei, L. C. Ciungu and A. B. Saeid, States on pseudo-BE algebras, J. Mult.-Valued Logic Soft Comput. 28 (2017), 591-618.

[28] A. Rezaei, A. B. Saeid and K. Y. S. Saber, On pseudo-CI algebras, Soft Computing 23(13) (2019), 4643-4654.

[29] A. Rezaei, A. B. Saeid and A. Walendziak, Some results on pseudo-Q algebras, Ann. Univ. Paedagog. Crac. Stud. Math. 16 (2017), 61-72.

[30] A. Rezaei, A. Walendziak and A. B. Saeid, Some remarks on commutative and pointed pseudo-CI algebras, Mathematica Aeterna 8(4) (2018), 269-277.

[31] S. Tanaka, A new class of algebras, Mathematical Seminar Notes 3 (1975), 37-43.

[32] A. Walendziak, Pseudo-BCH algebras, Discuss. Math. Gen. Algebra Appl. 35 (2015), 5-19.

[33] A. Walendziak, On branchwise commutative pseudo-BCH algebras, Ann. Univ. Mariae CurieSklodowska Sect. A LXXI(2) (2017), 79-89.

${ }^{1}$ Department of Mathematics, PAyAme NoOR University,

P. O. Box. 19395-3697, TEHRAN, IRAN

Email address: rezaei@pnu.ac.ir 